\title{
NDM-1 imported from India - first reported case in South Africa
}

\author{
C N Govind, ${ }^{1} \mathrm{MB}$ ChB, FCPath (SA) (Micro); K Moodley, ${ }^{1} \mathrm{MB}$ ChB, FCPath (SA) (Micro); A K Peer, ${ }^{1} \mathrm{MSc}, \mathrm{MB}$ ChB, MMed (Micro); \\ N Pillay, ${ }^{1}$ MSc; C Maske, ${ }^{2}$ MB ChB, PhD; C Wallis, ${ }^{2}$ MSc (Med), PhD (Med); R Viana, ${ }^{2}$ PhD (Med); A Chetty, ${ }^{3}$ MB ChB, MCFP (SA), MMed; \\ O Perovic, ${ }^{4}$ MD, DTM\&H, FCPath (SA) (Micro), MMed \\ ${ }^{1}$ Department of Clinical Microbiology, Lancet Laboratories, Durban, South Africa \\ ${ }^{2}$ Department of Molecular Biology, Lancet Laboratories, Johannesburg, South Africa \\ ${ }^{3}$ Family physician, Durban, South Africa \\ ${ }^{4}$ Centre for Opportunistic, Tropical and Hospital Infections at the National Institute of Communicable Diseases, Johannesburg, South Africa
}

Corresponding author: C N Govind (govindc@lancet.co.za)

\begin{abstract}
Carbapenem-resistant Enterobacteriaceae have been increasingly reported throughout the world. The first South African report of a New Delhi metallo-beta-lactamase was from Gauteng in August 2011. Despite maintaining a high degree of vigilance, the first such case was seen in KwaZulu-Natal almost a year later. Other cases have been unable to confirm a definite link to any other affected areas; this is the first case in South Africa showing this direct epidemiological link.

S Afr Med J 2013;103(7):476-478. DOI:10.7196/SAMJ.6593
\end{abstract}

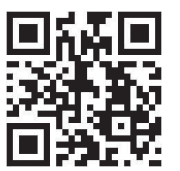

Carbapenems have long been a reliable last line of defence in the treatment of infections caused by antimicrobial-resistant Enterobacteriaceae. However, the emergence of resistance in Enterobacteriaceae mediated via carbapenemases is a major public health concern as these isolates show resistance to antibiotics commonly used to treat Gram-negative infections. ${ }^{[1]}$ Furthermore, these highly resistant strains have been shown to have the propensity to spread rapidly. ${ }^{[2]}$ A large variety of carbapenemases belonging to 3 classes of $\beta$-lactamases have been identified in Enterobacteriaceae: the Ambler class A, B and D $\beta$-lactamases. Carbapenem resistance as a result of Klebsiella pneumoniae carbapenemase (KPC; Class A), New Delhi metallo-beta-lactamase-1 (NDM-1; Class B) and OXAsubtypes (Class D) are thought to occur at low levels in Africa. ${ }^{[1]}$

The first case of NDM-1 carbapenem resistance in South Africa (SA) occurred in August $2011^{[3]}$ and was reported from a hospital in Johannesburg. The first documented case of NDM-1-producing Enterobacteriaecae in KwaZulu-Natal (KZN), SA, is reported here and documents a direct epidemiological link to the Indian subcontinent.

\section{Method and results Case report}

A 61-year-old woman presented to her general practitioner on 2 November 2012 for removal of a urinary catheter. She had been hospitalised in India from 21 to 24 October for management of a fractured femur. Antibiotics administered intra-operatively included cephalosporins and amikacin. Of note, urine submitted on admission to the hospital in India revealed no abnormalities. She was discharged on cefuroxime axetil $500 \mathrm{mg}$ twice daily for 1 week. She returned to South Africa on 1 November 2012 with a urinary catheter in situ (for ease of travel on the flight). On removal of the catheter the following day, her urine was noted to be turbid. She was, nevertheless, apyrexial and otherwise asymptomatic. There were no associated comorbid conditions. Urine dipstix revealed leucocyte esterase, prompting treatment with 3 doses of $3 \mathrm{~g}$ fosfomycin administered 2 days apart. A midstream urine specimen was sent to the laboratory.

\section{Laboratory investigations}

Urine microscopy revealed 10 red blood cells and 9 leucocytes/ high-power field. Culture revealed Enterobacter cloacae with the susceptibility pattern outlined in Table 1. Identification was confirmed using the automated Vitek 2 system (Biomerieux, Johannesburg, SA). Susceptibility testing was performed using the Kirby Bauer method and the Vitek 2 system. All susceptibility test results were interpreted using Clinical and Laboratory Standards Institute (CLSI) 2012 guidelines, ${ }^{[4]}$ except for tigecycline and colistin for which the 2012 European Committee on Antimicrobial Susceptibility Testing (EUCAST) breakpoints were used. ${ }^{[5]}$ Carbapenem and tigecycline minimum inhibitory concentrations (MICs) were determined by using E-tests (Biomerieux, South Africa) on Mueller-Hinton agar. Carbapenemase activity was suggested by the Vitek 2 expert system and confirmed by a positive modified Hodge test (CLSI guidelines 2012). A validated commercially available real-time PCR assay (Shanghai ZJ Bio-Tech) was used for the detection of New Delhi metallo-beta-lactamase 1 (NDM-1). DNA was extracted from culture, amplified and detected using a labelled primer/probe set. Inhibition was monitored by the use of an internal control. The isolate was confirmed NDM-1 positive. This result was verified by the National Institute for Communicable Diseases by multiplex real-time PCR using the LightCycler 480 Probes Master kit (Roche Diagnostics, USA). Repeat urine on 15 November 2012 showed no abnormalities and a negative culture.

\section{Discussion}

NDM-1 was first detected in an isolate of Klebsiella pneumoniae from a Swedish patient of Indian origin in $2008 .{ }^{[6]}$ It was subsequently detected in Enterobacteriaceae in India, Pakistan, the UK, USA, Canada, Japan, Brazil and Africa. ${ }^{[7,8,10]}$ The first documented report of NDM-1 in Enterobacteriacae in SA was from Gauteng in August $2011{ }^{[3]}$ The case presented here is the first documented case in KwaZulu-Natal, SA.

With increasing resistance among the Enterobacteriaceae to various classes of antimicrobial agents, carbapenems have formed 
Table 1. Antimicrobial susceptibility test results: Enterobacter cloacae NDM-1 positive

\begin{tabular}{|c|c|c|c|c|}
\hline \multirow[b]{3}{*}{ Antimicrobial agents } & \multicolumn{4}{|c|}{ Susceptibility test results } \\
\hline & Vitek 2 & Interpretation & E-test & Interpretation \\
\hline & $\operatorname{MIC}(\mu \mathrm{g} / \mathrm{ml})$ & $\mathbf{S} / \mathbf{I} / \mathbf{R}$ & $\operatorname{MIC}(\mu \mathrm{g} / \mathrm{ml})$ & $\mathbf{S} / \mathbf{I} / \mathbf{R}$ \\
\hline Ampicillin & $\geq 32$ & $\mathrm{R}$ & NT & \\
\hline Amoxycillin-clavulanic acid & $\geq 32$ & $\mathrm{R}$ & NT & \\
\hline Piperacillin-tazobactam & $\geq 128$ & $\mathrm{R}$ & NT & \\
\hline Cefepime & $\geq 64$ & $\mathrm{R}$ & NT & \\
\hline Ceftriaxone & $\geq 64$ & $\mathrm{R}$ & NT & \\
\hline Cefoxitin & $\geq 64$ & $\mathrm{R}$ & NT & \\
\hline Cefuroxime & $\geq 64$ & $\mathrm{R}$ & NT & \\
\hline Ceftazidime & $\geq 64$ & $\mathrm{R}$ & NT & \\
\hline Doripenem & NT & & 16 & $\mathrm{R}$ \\
\hline Ertapenem & $\geq 8$ & $\mathrm{R}$ & NT & \\
\hline Imipenem & $\geq 16$ & $\mathrm{R}$ & 12 & $\mathrm{R}$ \\
\hline Meropenem & $\geq 16$ & $\mathrm{R}$ & 24 & $\mathrm{R}$ \\
\hline Gentamicin & $\geq 16$ & $\mathrm{R}$ & NT & \\
\hline Amikacin & $\geq 32$ & $\mathrm{R}$ & NT & \\
\hline Tigecycline ${ }^{*}$ & NT & & 0.5 & S \\
\hline Ciprofloxacin & $\geq 4$ & $\mathrm{R}$ & NT & \\
\hline Cotrimoxazole & $\geq 320$ & $\mathrm{R}$ & NT & \\
\hline Fosfomycin $^{\dagger}$ & NT & & NT & \\
\hline Colistin ${ }^{\star}$ & $\leq 2$ & S & NT & \\
\hline
\end{tabular}

the backbone of treatment. The emergence of novel $\beta$-lactamases with direct carbapenem-hydrolysing activity has contributed to an increased prevalence of carbapenem-resistant Enterobacteriaceae (CRE). These are usually accompanied by resistance mechanisms to other classes of antibiotics, thereby posing an additional therapeutic challenge. ${ }^{[8]}$ CREs are a significant threat for a number of reasons: the frequency with which they cause infections, the high mortality with which they are associated ${ }^{[9]}$ and the potential for their widespread transmission via mobile genetic elements. ${ }^{[8]}$ The NDM-1 gene found on a plasmid is highly transmissible to other bacteria. NDM-1positive bacteria are capable of colonising the gastro-intestinal tract of humans for prolonged periods and are spread through contamination of water and environmental surfaces. ${ }^{[6]}$ The ease and frequency of international travel has contributed additionally to rapid global dissemination.

Current data suggest that recent hospitalisation in, and perhaps even travel to, the Indian subcontinent be identified as a risk factor for colonisation with the NDM-1-producing strain, ${ }^{[8,11]}$ recognising that medical care is often sought by individuals visiting relatives in India and Pakistan. A recent case of NDM-1-producing Providencia rettgeri in an Australian who received elective plastic surgery in India illustrates this phenomenon. ${ }^{[10]}$ Despite these factors, there are very few cases with a confirmed epidemiological link. ${ }^{[11]}$ In the editorial by Coetzee et al., on the emergence of CREs in SA, the origin of the NDM-1s was not determined. ${ }^{[12]}$ In the light of the KZN case, strong consideration should be given to screening patients (testing of rectal swabs for detection of NDM-1 carriage) with a history of recent hospitalisation in the Indian subcontinent who present for medical care. Given that NDM-1 is a globally emerging phenomenon, a case might be made for routine screening for CRE colonisation in patients after recent hospitalisation overseas and in those recently admitted to hospitals in Gauteng and Cape Town, since, following the first reported case in Gauteng, that outbreak has been ongoing.

It is also important to note that other molecular mechanisms of carbapenem resistance have been reported in SA, viz. OXA-48, KPC, VIM and GESs. ${ }^{[13]}$ Early recognition and reporting provides an opportunity to establish national measures to prevent such isolates becoming endemic in healthcare settings. Owing to the limited therapeutic options available, early identification of CREs and timely isolation and infection control measures are key to curtailing the spread of these organisms. Concerns about carbapenemases justify a high index of suspicion in diagnostic laboratories and the routine screening of all clinically significant Enterobacteriaceae for susceptibility to carbapenem agents. Rapid detection is essential for patient management and prevention of further transmission, with the aim of limiting the public health impact of these extremely drugresistant strains.

\section{Conclusion}

To our knowledge, this is the first documented case of an NDM-1 Enterobacteriacea in KZN and the first case in SA where a direct epidemiological link to the Indian subcontinent has been established. Since the detection of this isolate, other cases in several hospitals have been identified. Ongoing vigilance and strict infection control measures need to be maintained. 


\section{References}

1. Chen LF, Anderson DJ, Paterson DL. Overview of the epidemiology and the threat of Klebsiella pneumonic carbapenemases (KPC) resistance. Infect Drug Resist 2012;5:133-141. [http://dx.doi.org/10.2147/IDR.S26613] 2. Kumarasamy K, Thirunarayan MA, Krishnan P. Coexistence of blaOXA-23 with blaNDM-1 and armA in clinical isolates of Acinetobacter baumanii from India. J Antimicrob Chemother 2010;65:2253-2254 [http://dx.doi.org/10.1093/jac/dkq273]

3. W Lowman, C Sriruttan, T Nana, et al. NDM-1 has arrived: First report of a carbapenem resistance mechanism in South Africa. S Afr Med J 2011;101(12):873-875.

4. Clinical Laboratory Standards Institute. Performance Standards for Antimicrobial Susceptibility Testing; Twenty-second informational supplement. CLSI document M100-S22. Wayne, PA, USA: CLSI, 2012. 5. European Committee on Antimicrobial Susceptibility Testing (Eucast) documents. Document version 5. European Committee on Antimicrobial Susceptibility Testing (Eucast) documents. Document version

6. Yong D, Toleman MA, Giske CG, et al. Characterization of a new metallo-beta-lactamase gene, bla 6. Yong D, Toleman MA, Giske CG, et al. Characterization of a new metallo-beta-lactamase gene, bla
(NDM-1), and a novel erythromycin esterase gene carried on a unique genetic structure in Klebsiella (NDM-1), and a novel erythromycin esterase gene carried on a unique genetic structure in Klebsiella
pneumoniae sequence type 14 from India. Antimicrob Agents Chemother 2009;53:5046-5054. [http:// pneumoniae sequence type 14 from
dx.doi.org/10.1128/AAC.00774-09]

7. Poirel L, Revathi G, Bernabeu S, Nordmann P. Detection of NDM-1-producing Klebsiella pneumoniae in Kenya. Antimicrob Agents Chemother 2011;55:934-936. [http://dx.doi.org/10.1128/AAC.01247-10]
8. Kumarasamy KK, Toleman MA, Walsh TR, et al. Emergence of a new antibiotic resistance mechanism India, Pakistan, and the UK: A molecular, biological, and epidemiological study. Lancet Infect Dis 2010;10:597-602. [http://dx.doi.org/10.1016/S1473-3099(10)70143-2]

9. Schwaber MJ, Klarfeld-Lidji S, Navon-Venezia S, Schwartz D, Leavitt A, Carmeli Y. Predictors of carbapenemresistant Klebsiella pneumoniae acquisition among hospitalized adults and effect of acquisition on mortality. Antimicrob Agents Chemother 2008;52:1028-1033. [http://dx.doi.org/10.1128/AAC.01020-07]

10. Walsh T. New Delhi metallo- $\beta$-lactamase-1: Detection and prevention. CMAJ 2011;183(11):1240-1241. [http://dx.doi.org/10.1503/cmaj.111100]

11. Borgia S, Lastovetska O, Richardson D, et al. Outbreak of carbapenem-resistant Enterobacteriacae- containing blaNDM-1, Ontario, Canada. Clin Infect Dis 2012;55:e109-117. [http://dx.doi.org/10.1093/cid/cis737]

12. Coetzee J, Brink A. The emergence of carbapenem resistance in Enterobacteriaceae in South Africa. S Afr J Epidemiol Infect 2011;26(4):239-240.

13. Brink A, Coetzee I. The spread of carbapenem-resistant Enterobacteriacea in South Africa: Risk factors for acquisition and prevention. S Afr Med J 2012;102(7):599-601.

Accepted 24 January 2013 